\section{GW23-e2316 EFFECTS OF ROOT OF HERBACEOUS PEONY ON LIPID METABOLISIM OF RHEUMATOID ARTHRITIS PATIENTS}

doi:10.1136/heartjnl-2012-302920ac.2

XJ Hou, YL zhu, XJ Hou. Dong Fang Hospital Affiliated to Beijing University of

Traditional Chinese Medicine

Objectives Rheumatoid arthritis can cause multi-joints damage and ultimately leads to joint function obstacle. There is shortened life expectancy with RA patients, cardiovascular disease is considered to be the primary cause of death in patients with RA, which is an independent risk factor for cardiovascular disease. The coronary atherosclerotic heart disease risk of RA patients is higher, and which maybe related with the influence of RA on the lipid metabolism. Traditional Chinese medicine Root of herbaceous peony can decrease the blood fat, which has been used in the treatment of fatty liver, and the root of herbaceous peony glucoside (TGP) capsule is used in the treatment of rheumatoid arthritis. This report is to observe the effects of TGP on RA patients lipid levels .

Methods Active RA patients were selected in accordance with the revised 1987 rheumatism rheumatoid arthritis classification standard, and the DAS28 score more than 3.2. Exclusion criteria for the application of any influence blood lipid drug cases. For men in patients 12 cases, female 40 cases. The patient before treatment ( 0 month) and after treatment ( 6 months after treatment) were determined the lipid levels (TC, TG, LDL-C, ApoA, ApoB), c-reactive protein, blood sedimentation and DAS28 score. Patients venous were determinated with an empty stomach, automatic biochemistry analyser determine were method was be used, and the immune method to determine the c-reactive protein; all patients used methotrexate $10 \mathrm{mg} /$ week, or Leflunomide $10 \mathrm{mg} /$ day. Thirty cases were randomly selected to use total root of herbaceous peony glucoside in capsule $(0.6 \mathrm{~g}$, three times a day). Finally five patients were lost to follow-up, one case of root of herbaceous peony group (BSG), and four cases of conventional treatment group (CGZ).

Results There is no significant difference of the blood lipid levels and DAS28 score, ESR, c-reactive protein level between the two groups $(p>0.05)$. Treatment after 6 months, two groups of

patients (CGZ and BSZ) of blood lipid levels were TC (3.24; 3.17); TG (1.24; 1.31); LDL-C (2.14; 2.25); blood lipid levels of BSZ decreased than those before treatment, and TC and LDL-C decreased significantly $(p<0.05)$; DAS28 score of two groups after 6 months treatment decreased than those before the treatment $(p<0.05 ; p<0.05)$, DAS28 of BSZ decrease more than that of CGZ, but there was no statistical difference ( $p>0.05$ );

Conclusions Active RA patients often appear high TC and high LDL-C, which is related with high cardiovascular events, and is related with chronic inflammation and continued immune disorders. Root of herbaceous peony glucoside can be used to treat the RA, and also can decrease the blood fat level of the RA patients. To determine if it can reduce cardiovascular events of the RA patient, the longer follow-up will still be needed to do. 